\title{
Application of the C-Credibility Measure
}

\author{
Marija PAUNOVIĆ, Nebojša RALEVIĆ, Vladimir GAJOVIĆ
}

\begin{abstract}
The complex logistics process involves numerous dynamic uncertainties and internal risks. Those risks alone or in interaction with other groups of risks can affect the entire system. This paper proposes the application of the new measure, c-credibility measure to the complex system such as logistic process. For this purpose, fuzzy logic system for risk assessments in logistic process is proposed. C-credibility selection through ranking is implemented in the simulation process with $c r_{h}$ membership function definition on the fuzzy variable to calculate credibility value. Numerical analysis of the proposed approach with obtained results and the related discussion is presented.
\end{abstract}

Keywords: c-credibility measure; fuzzy logic; simulation

\section{INTRODUCTION}

With the widespread use of the supply chain on the global level, logistics processes are becoming increasingly prominent as its integral component. The complex logistics process involves numerous dynamic uncertainties and internal risks. The notion of uncertainty implies that there is a possible spectrum of events that can happen in the future, however without indications that they will really happen or have a relative effect. The uncertainty is also described as the absence of information in a decisionmaking process and implies the assessment of a particular situation, alternative solutions, possible results and consequences, etc.

Traditionally, probability theory handles uncertainty, but different modern techniques, evolved from fuzzy sets theory, Dempster-Shafer theory, approximate reasoning, possibility theory and credibility theory, are being increasingly used.

In the research [3], three different models are presented. Two of them are based on the fuzzy techniques and simulation that have treated the same logistic process for the purpose of risk assessment systems. Fuzzy logic models with simulation in marine system are presented in [13] and in traffic system in [2]. There are many hybrid models using a combination of some of the techniques mentioned [14, 15]. Simulations implemented in the models make it possible to obtain different results for different scenarios and to define the changes that are necessary to meliorate the performance of the observed system.

Another version of the credibility theory in the fuzzy environment, as a part of mathematics for examined fuzzy events, was formulated in [8]. The credibility measure [7, 9] is presented as no additive measure with self-duality property, as an average of possibility and necessity measures. In the classical theory of credibility, the main task is to find weight of measures. As the weight of measures was predetermined, Ralevic and Paunovic [12] came up with the idea to generalize credibility measure and that is achieved with so called c-credibility measure.

In recent studies, credibility in fuzzy environment has been used in various applications. A fuzzy optimization model is proposed in [16] based on credibility theory for electric vehicle routing problem with time windows and recharging stations. Wang et. al [14] in their study selected to measure the safety degree under the fuzzy inputs and binary state with credibility measure in the aircraft icing safety analysis method. Pourrahmani et al. [11] implemented fuzzy credibility in the genetic algorithm to optimize the evacuation problem and applied it on the concrete transportation network.

Bearing in mind the aforementioned and the fact that the c-credibility is a new measure, this paper proposes the application of this measure to the complex system such as logistic process. For this purpose, a method based on fuzzy logic for risk assesments in logistic process is proposed. Ccredibility ranking is implemented in the simulation process with membership function definition on the fuzzy variable to calculate credibility value. Further, keeping in mind that it is much easier for system users to estimate parameters on the same scale, the model uses individual values of uniform distributed parameters in the same domain interval for each parameter. Besides that, proposed procedure in iteration considers the influence of the simulated parameter.

The rest of the paper is organised as follows: the concept, definition and properties of the c-credibility measure are introduced in section 2. Proposed approach and procedure with the problem formulation is presented in section 3. Computational analysis of the proposed approach with obtained results and the related discussion is presented in section 4 . We conclude our paper with a summary of the main findings.

\section{PRELIMINARIES}

Aggregation of information takes a significant place in many knowledge-based systems, where aggregation of data or values is needed. In general, it can be said that by aggregation, different parts of information from different sources are used at the same time, in order to make a conclusion or a decision.

Aggregation function [5] is defined by a function $h:[0,1]^{n} \rightarrow[0,1]$ such that

h1) $h(0, \ldots, 0)=0$ and $h(1, \ldots, 1)=1$,

h2) $h\left(v_{1}, \ldots, v_{n}\right) \leq h\left(\omega_{1}, \ldots, \omega_{n}\right)$ such that $v_{i} \leq \omega_{i}$

h3) $h(v)=v$ for all $v \in[0,1]$. 
Condition $h(0, \ldots, 0)=0$ means that if we consider only completely poor, inaccurate or unsatisfactory criteria, overall aggregation must also be completely poor, incorrect or unsatisfactory. The interpretation of condition $h(1, \ldots, 1)=1$, is that if we only look at the completely correct or completely satisfactory criteria, then the overall aggregation must also be completely correct or completely satisfactory.

When applied to fuzzy sets $E_{1}, E_{2}, \ldots, E_{n}$ defined on $X$, function $h$ creates an aggregate fuzzy set A by operating on the membership grades of these sets for each $\omega \in X$.

Thus, $A(\omega)=h\left(E_{1}(\omega), \mathrm{E}_{2}(\omega), \ldots, \mathrm{E}_{n}(\omega)\right)$ for each $\omega \in X$.

Aggregation function is commutative if $h$ is a symmetric function on all its arguments, which means that the aggregated fuzzy sets are equally significant and the order of the arguments is irrelevant to the result. If this assumption is not justified in some applications, the symmetry property is rejected.

Some examples of aggregation functions are weighted arithmetic mean, quasi-arithmetic mean, root-power mean. More information about aggregation functions can be found in, for example Dubois and Prade [1] or Grabish, Marichal, Mesiar and Pap [4 ].

Possibility and necessity measures, as monotone and semi continuous measures, are being increasingly applied in real problems.

Set function nec defined on $(X, \Sigma)$ is a fuzzy measure if $\operatorname{nec}\left(\bigcap_{j \in J} V_{j}\right)=\inf _{j \in J} n e c\left(V_{j}\right)$, or for all $\left\{V_{j} \mid j \in J\right\}$ in $\Sigma$ such that $\bigcap_{j \in J} V_{j} \in \Sigma$, where $J$ is an arbitrary index set.

Set function pos defined on $(X, \Sigma)$ is a fuzzy measure if $\operatorname{pos}\left(\bigcup_{j \in J} V_{j}\right)=\sup _{j \in J} \operatorname{pos}\left(V_{j}\right)$, or for all $\left\{V_{j} \mid j \in J\right\}$ in $\Sigma$ such that $\bigcup_{j \in J} V_{j} \in \Sigma$, where $J$ is an arbitrary index set.

We have that $n e c(V)=1-\operatorname{pos}(\bar{V})$.

As this is a new measure, the main definitions and theorems will be presented in this part of the paper. New measure of uncertainty called c-credibility measure is defined in [12] as follow.

Definition 1 . Let $c:[0,1] \rightarrow[0,1]$ be an involutive fuzzy complement, whose equilibrium is $\xi$. The c-credibility measure on $\mathrm{X}$ (determined by $\mathrm{c}$ ) is a set function $\mathrm{cr}: P(X) \rightarrow[0,1]$ such that

Ax1) $\operatorname{cr}(\varnothing)=0$;

Ax2) $\left(\forall V, V^{\prime} \in P(X)\right) \mathrm{V} \subset V^{\prime} \Rightarrow \operatorname{cr}(V) \leq c r\left(V^{\prime}\right)$;

Ax3) $(\forall V \in P(X)) c r(\bar{V})=c(c r(V))$;

Ax4) $c r\left(\bigcup_{j \in J} V_{j}\right)=\underset{j \in J}{\sup c r}\left(V_{j}\right)$, for any sets $V_{j} \in P(X), j \in J$, for which $\sup _{j \in J} \operatorname{cr}\left(V_{j}\right)<\xi$, where $J$ is an arbitrary index set.

The triplet $(X, P(X), c r)$ is called a $\mathrm{c}-$ credibility space.

Example 1. For set function $\operatorname{cr}: P(X) \rightarrow[0,1]$, $\Omega=\left\{\omega_{1}, \omega_{2}, \omega_{3}, \omega_{4}\right\}$, defined with $\operatorname{cr}(X)=1, \operatorname{cr}(\varnothing)=0$, $\operatorname{cr}\left\{\omega_{1}\right\}=0.9, \operatorname{cr}\left\{\omega_{2}\right\}=0.2, \operatorname{cr}\left\{\omega_{3}\right\}=0.3, \operatorname{cr}\left\{\omega_{4}\right\}=0.4$, $\operatorname{cr}\left(\left\{\omega_{1}, \omega_{2}\right\}\right)=0 . \overline{18}, \operatorname{cr}\left(\left\{\omega_{1}, \omega_{3}\right\}\right)=0 . \overline{18}$, $\operatorname{cr}\left(\left\{\omega_{1}, \omega_{4}\right\}\right)=0.125, \operatorname{cr}\left(\left\{\omega_{2}, \omega_{3}\right\}\right)=0.3$, $\operatorname{cr}\left(\left\{\omega_{2}, \omega_{4}\right\}\right)=0.4, \operatorname{cr}\left(\left\{\omega_{3}, \omega_{4}\right\}\right)=0.4$, $\operatorname{cr}\left(\left\{\omega_{1}, \omega_{2}, \omega_{3}\right\}\right)=0 . \overline{18}, \operatorname{cr}\left(\left\{\omega_{1}, \omega_{2}, \omega_{4}\right\}\right)=0.125$, $\operatorname{cr}\left(\left\{\omega_{1}, \omega_{3}, \omega_{4}\right\}\right)=0 . \overline{142857}, \operatorname{cr}\left(\left\{\omega_{2}, \omega_{3}, \omega_{4}\right\}\right)=0.4$, is a c-credibility measure with respect to complement $c(\omega)=\frac{1-\omega}{1+2 \omega}$.

If there are more than two singletons whose measure of c-credibility is different from zero, the c-credibility measure cannot be additive. We will assume that the credibility of each singleton is specified.

In the following we give the Extension Theorem of ccredibility.

Let $X$ be an empty set and $c r: P(X) \rightarrow[0,1]$ $\mathrm{c}-$ credibility, it is

$$
\begin{aligned}
& \sup _{\omega \in X} \operatorname{cr}(\{\omega\}) \geq \xi \\
& \operatorname{cr}\left(\left\{\omega^{*}\right\}\right) \geq \xi \Rightarrow \sup _{\omega \neq \omega^{*}} \operatorname{cr}(\{\omega\})=c(\operatorname{cr}(\{\omega\})) .
\end{aligned}
$$

Next theorem determines the c-credibility measure in numerical value based on credibility value of each singleton set. The credibility extension theorem gives a sufficient condition for the c-credibility measure.

Theorem 1. Let $c:[0,1] \rightarrow[0,1]$ be an involutive fuzzy complement, with equilibrium $\xi$, and $c r:\{\{\omega\} \mid \omega \in X\} \rightarrow R_{0}^{+}, X \neq \varnothing$ a set function satisfying (1) and (2). Then $c r$ has a unique extension on the $\mathrm{c}-$ credibility measure $c r: P(X) \rightarrow R_{0}^{+}$then

$\operatorname{cr}(V)=\left\{\begin{array}{cl}\sup _{\omega \in V} \operatorname{cr}(\{\omega\}), & \sup _{\omega \in V} \operatorname{cr}(\{\omega\})<\xi \\ c\left(\sup _{\omega \in V} \operatorname{cr}(\{\omega\})\right), & \sup _{\omega \in V} \operatorname{cr}(\{\omega\}) \geq \xi\end{array}\right.$

for non empty set $V$, and $\operatorname{cr}(\varnothing)=0$.

Credibility of a Fuzzy Events

Let $X$ be a TFN on $(X, P(X)$,pos), with the membership function 
$\mu(\omega)=\left\{\begin{aligned} \frac{\omega-l}{m-l}, & l<\omega<m \\ \frac{r-\omega}{r-m}, & m<\omega<r \\ 0, & \omega \leq l \vee r \leq \omega\end{aligned}\right.$

Possibility of a fuzzy event $\{X \leq \omega\}$ is described with $\operatorname{pos}(\{X \leq \omega\})=\sup _{z \leq \omega} \mu(z)$ and for TFN number it is

$\operatorname{pos}(\{X \leq \omega\})=\left\{\begin{aligned} 0, & \omega \leq l \\ \frac{v-l}{m-l}, & l<\omega<r \\ 1, & r \leq \omega\end{aligned}\right.$

Necessity of a fuzzy event $\{X \leq \omega\}$ is described as

$$
\begin{aligned}
& \operatorname{nec}(\{X \leq \omega\})=1-\operatorname{pos}(\{X>\omega\})=1-\sup _{z>\omega} \mu(z) \\
& =\left\{\begin{aligned}
0, & \omega \leq m \\
\frac{\omega-m}{r-m}, & m<\omega<r \\
1, & r \leq \omega
\end{aligned}\right.
\end{aligned}
$$

In [12] is defined the $c$-credibility in the relation with aggregation function $h$ with

$$
c r_{h}(E)=h(\operatorname{pos}(E), \operatorname{nec}(E))
$$

Which implies

$$
\begin{aligned}
& c r_{h}(\{X \leq \omega\})=h(\operatorname{pos}(\{X \leq \omega\}), \operatorname{nec}(\{X \leq \omega\}))= \\
& =\left\{\begin{aligned}
0, & \omega \leq l \\
h\left(\frac{\omega-l}{m-l}, 0\right), & l<\omega \leq m \\
h\left(1, \frac{\omega-m}{r-m}\right), & m<\omega<r \\
1, & r \leq \omega
\end{aligned}\right.
\end{aligned}
$$

The fuzzy event holds, for credibility 1 , and fails for credibility value 0 .

\section{PROPOSED APPROACH}

In practice, many unknown and rare situations and problems can occur that cannot be predicted or prevented, but can affect the operation of the observed system. For these reasons, procedure which directly adopts expertise from fuzzy matrices and simulates variables ranked by cmeasure credibility, allows minimizing the subjectivity of expert judgment by fuzzy logic, in some circumstances can be a better solution compared to others.

In this paper, we modified the $2 \mathrm{sFL}$ model presented in [10]. Two very significant changes were introduced. First, c-credibility theorem uses the membership function definition of the fuzzy variable to calculate its credibility value. C-credibility ranking is implemented in the simulation process. Second, keeping in mind that it is much easier for system users to estimate parameters on the same scale, the model uses individual values of uniform distributed parameters in the domain interval $[0 ; 100]$ for each element of risk. Besides that, procedure in iteration considers the influence of the simulated parameter.

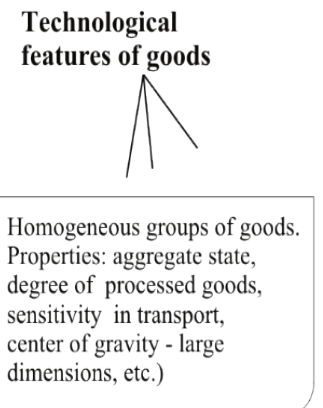

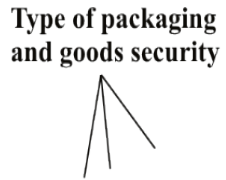

Type of packaging: characteristics of packaging and technology, palletization, packaging standards, container type etc.

Security of goods in transit and during storage.

Preventive measures.
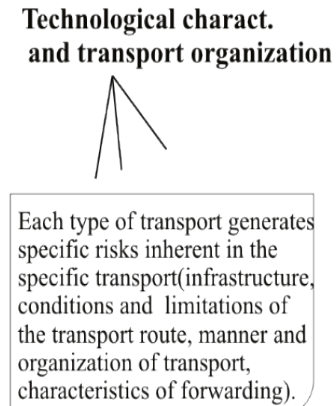

Figure 1 Parameters description

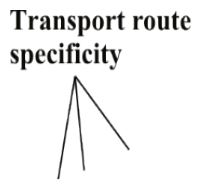

Physical distance of sender and receiver, transport and traffic infrastructure, area and

destination, climate conditions, political conditions, terms and restrictions of specific transport and other.
Other logistic parameters

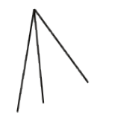

Manipulation risks, level of technology and quality of transfer services and the duration of transfer,interruption of transport warehousing, administrative restrictions and certain national regulations, etc.
Using fuzzy techniques, one can easily estimate the uncertain element based on expert opinion, experience or historical information. The first task is to establish input parameters of the logistic process. In many researches, there is a good preparation of fuzzy variables related to the observed problem. In order to illustrate the use of ccredibility in the fuzzy environment, we used data from work [3], where authors analysed the total risk in logistics processes. Experts from domain of transportation technologies and logistic processes are studying such problem.

Risk elements are classified into five data sets (basic risk parameters, V1, V2, V3, V4, V5), illustrated in Fig. 1.
The identified risk elements contain a number of influential parameters that, individually or together with other parameters, determine the overall risk of concrete logistics processes.

For example, second group of parameters, type of packaging, is closely connected to technological features of goods. The risk level is highly affected by goods protection (packaging, palletization, containerization, etc) as well as security during transport, storage etc. Packaging is an integral part of goods, and the selection of optimal packaging may minimize the risk and form one part of preventive actions. Same type of goods can be packed in different types of commercial packaging that may be of 
different material and different protection in respect of external conditions, which significantly affects the risk in transport. Goods palletization implies bringing packaging units together onto a pallete what makes for additional protection of goods. Apart from optimal packaging, the risk is highly affected by transport containerization. One of many advantages of goods containerization is the fact that the exposure to external, static and dynamic conditions, weather conditions, theft risk etc is significantly minimized. The basic role of optimal security is protection of goods from damages that most often occur due to different dynamic influences during transport and handling. Further, different types of transport have specific risks characteristic of the given transport (organization). Particular types of transport last significantly longer than some others, which has a significant impact on the risk exposure duration. By rule, the risks in combined transport are increased due to the fact that the goods are several times reloaded, withheld, warehoused at intermediate points etc, so the organization of transport (third group of data) is closely linked to packaging and security. In case of transport organized by forwarders or agents, there are certain limitations on risk control but also a possibility of indemnity of possible losses based on legal and contractual obligations towards carriers or forwarders for damages to goods.

At these complex issues experts looked comprehensively and determined the pairwise comparison of different criteria relevant to the overall goal. Expert's assessments are transformed in fuzzy numbers and represent input data.

In the simulation process fuzzy variables are ranked using c-credibility measure. The ranking procedure is described below.

Credibility procedure. Credibility measures the degree of confidence given to a specific data set. Credibility theory aims at efficiently combining information from diverse sources: past and current data, individual and collective data, etc. [6]

The membership functions of the fuzzy variables need to be estimated to assure that their capability representing the data features efficiently. This analysis helps in giving the credibility to the, for example, expertise, credibility to the whole fuzzy system, its parts, etc. Using the ccredibility inversion theorem, credibility value can be calculated based on assumption that the fuzzy variable belongs to an event $E$ and calculating the aggregation of the possibility that the event happens and the necessity of the event complement has happened too. The $c$-credibility in the relation with aggregation function $h$ of a fuzzy event is used in ranking process. Ranking procedure hires each fuzzy variable $\mathrm{z}$ and arbitrary chooses a fuzzy number which is necessary to establish assumption about event $E$. The process generates members of event $E$ and generated possibility, $\sup \mu(z)$ of these numbers in the event $E$ and $z \leq x$

its complement, $1-\sup \mu(z)$. On the two groups of data $(E$ $z \leq x$

membership or $c(E)$ membership), the aggregate of the supreme function of the membership degrees of those two groups is calculated which is denoted as the c-credibility value.
From the property that pos measure on the power set of the finite set $X=\left\{\omega_{1}, \omega_{2}, \ldots, \omega_{n}\right\}$ can be uniquely determined from the distribution function of the possibility function $\quad r: X \rightarrow[0,1]$ using formula $\operatorname{pos}(E)=\max _{\omega \in E} r(\omega), \quad$ knowing the values of the singleton as $\operatorname{pos}(E)=\max \left(\operatorname{pos}\left(\omega_{1}\right), \ldots, \operatorname{pos}\left(\omega_{n}\right)\right)$.

The values of $\operatorname{pos}(E)$ and thus nec $(E)$, for some sets of existing data can be taken from that dataset, while the values for other sets, especially singletons (that are missing) can be simulated.

According to the definitions and properties given in section 2 , the possibility, necessity and credibility for this fuzzy variable $E$ can be derived as follows. For FTN that represents low risk (see Fig.2), c-credibility function in fuzzy environment is:

$c r_{h}(\{X \leq \omega\})= \begin{cases}0, & \omega \leq 5 \\ h\left(\frac{\omega-5}{20}, 0\right), & 5<\omega \leq 25 \\ h\left(1, \frac{45-\omega}{20}\right), & 25<\omega \leq 45 \\ 1, & \omega>45\end{cases}$

c-credibility function in fuzzy environment for medium risk is:

$c r_{h}(\{X \leq \omega\})= \begin{cases}0, & \omega \leq 30 \\ h\left(\frac{\omega-30}{20}, 0\right), & 30<\omega \leq 50 \\ h\left(1, \frac{70-\omega}{20}\right), & 50<\omega \leq 70 \\ 1, & 70 \leq \omega\end{cases}$

Similar for all other levels of risk.
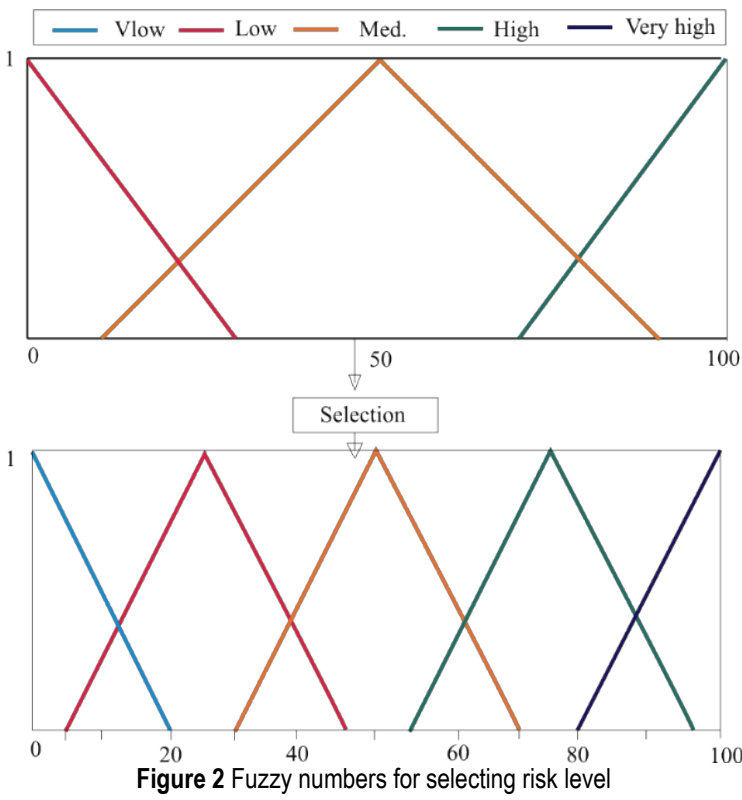

We can choose different aggregation functions that are optimum for our problem. 
Simulation evaluation system. By simulating individual values of uniformly distributed parameters over a domain interval $[0 ; 100]$ for each risk parameter, the individual impact of each risk parameter relative to the total risk can be determined. The simulation procedure is performed by changing one parameter (which shows the weight rating of each element of risk) according to the simulation experiment and considering the impact on the overall risk. After one iteration, the impact of each risk element in the range: 0 and 100 is analyzed by a simulation experiment, while the other parameters retain values obtained on the basis of a uniform distribution. After one simulation experiment, the next parameter changes until all the parameters in the first simulation of the total risk are changed. Procedure is similar with the recommendation document, Guide to the Expression of Uncertainty in Measurement, JCGM 101:2008.

Output data is derived by if-then rules which are implemented in the system, where linguistic values are levels of risk. In this way, the data presented quantitatively and qualitatively are united uniformly.

For each element $i$, a random number $\xi$ in the interval of triangular fuzzy number $\left[\omega_{i j}, \omega_{i j}\right]$ is generated with appropriate membership function $\mu_{\xi}$. In other words, procedure generates fuzzy numbers that belong to event $\mathrm{E}$, and calculates the degree of number to event $E$ or to complement event $E$. We get two sets of results, and then by applying formula (3) calculate the c-credibility value. The most credible elements are chosen based on threshold $\varepsilon$. The process of simulation of the parameters is repeated $\mathrm{M}$ times. The average results of the selected values, for each parameter, are calculated and represent model output.

\section{RESULTS OF THE MODEL}

The expert team of engineers related to the transport and logistic identified 5 data sets and observed their influential parameters and subparameters (Fig. 1).

Input parameters are triangular fuzzy numbers

$A=\left(e_{i j}\right)_{5 \times 5}, e_{i j}=\left(\omega_{i j}, \omega_{i j}, \omega_{i j}\right)$, as is presented in Tab. 1.

Fuzzy system is built using a fuzzy inference system at

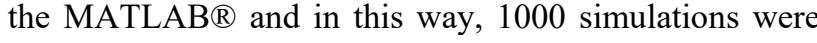
performed. The above procedure enables the testing of the output values (or impacts) of each individual risk parameter in relation with total risk. Each parameter is simulated as an independent variable with uniform distribution, $X \sim U(0,100)$. In the simulation process, parameters with credible values $\geq 0,7$ are selected for further calculation (Tab. 2). Average on the level of each parameter is calculated and presented in Tab. 3 .

Table 1 System input values

\begin{tabular}{|c|c|c|c|}
\hline Risk/Variable & $L$ & $M$ & $H$ \\
\hline V1 & $(0,0,13.22)$ & $(4.41,22.03,39.65)$ & $(30.84,44.05,44.05)$ \\
\hline V2 & $(0,0,7.80)$ & $(2.60,13.00,34.40)$ & $(18.20,26.00,26.00)$ \\
\hline V3 & $(0,0,2.39)$ & $(0.80,3.40,7.16)$ & $(5.57,7.95,7.95)$ \\
\hline V4 & $(0,0,2.63)$ & $(0.88,4.38,7.88)$ & $(6.13,8.75,8.75)$ \\
\hline V5 & $(0,0,3.98)$ & $(1.33,6.63,11.93)$ & $(9.28,13.25,13.25)$ \\
\hline
\end{tabular}

Table 2 Part of the simulation evaluation - selected outputs using credibility

\begin{tabular}{|c|c|c|c|c|c|c|c|c|c|c|c|c|c|c|c|}
\hline V1 & & V3 & V4 & V5 & utput & $\mathrm{V} 1=0$ & $\mathrm{~V} 1=100$ & $V 2=0$ & $V 2=100$ & $V 3=0$ & $V 3=100$ & $V 4=0$ & $V 4=100$ & & \\
\hline 99 & 85 & 5 & 9 & & & 207 & & & & & & & & & \\
\hline 0 & & & & & & & & & & & & & & & \\
\hline 50 & & & & & & & & & & & & & & & \\
\hline 91 & & & 15 & & & & & & & & & & & & \\
\hline 38 & & & & & & & & & & & & & & & \\
\hline 96 & & & & & & & & & & & & & & & \\
\hline 66 & & & & & & & & & & & & & & & \\
\hline 1 & 4 & & & & & & & & & & & & & & \\
\hline 35 & & & & & & & & & & & & & & & \\
\hline 60 & & & & & & & & & & & & & & & \\
\hline 35 & & & & & & & & & & & & & & & \\
\hline 50 & 19 & & & & & & & & & & & & & & \\
\hline 16 & & & & & & & & & & & & & & & \\
\hline 33 & 64 & & & & & & & & & & & & & & \\
\hline 52 & & & & & & & 85,8936 & & & & & & & & \\
\hline 29 & 31 & & & & & & & & & & & & & & \\
\hline 48 & & & & & & & & & & & & & & & \\
\hline 46 & 0 & 13 & 22 & 44 & 21,2517 & 8,3333 & 54,9172 & 21,2517 & 63,2164 & 21,2517 & 25,0000 & 11,7251 & 25,0000 & 14,4737 & 29,9172 \\
\hline
\end{tabular}

Table 3 Fuzzy system output

\begin{tabular}{|c|c|c|c|c|c|}
\hline Parameter & V1 & V2 & V3 & V4 & V5 \\
\hline Value & 0,45568 & 0,2615 & 0,06643 & 0,08208 & 0,1343 \\
\hline
\end{tabular}

According to the result, the highest value in the logistic process evaluation has the first group of parameters, which include technological features of goods (V1) with $45,57 \%$ of influence. In the second place is packaging and security of goods (V2), with $26,15 \%$. Other parameters V5 (handling technology, quality of reloading services as well as duration, specific characteristics of local environment, technological principles of storage, climate factors etc) has 
impact of $13,43 \%$. Both V3 and V4 have relatively small influence on the total risk, respectively $6,64 \%$ and $8,21 \%$.

Comparing the results obtained on the same data set using a different model [3], the result shows a $6 \%$ difference for the most influencing parameter and about $3 \%$ for the V2 parameter. Values of the other parameters are almost similar.

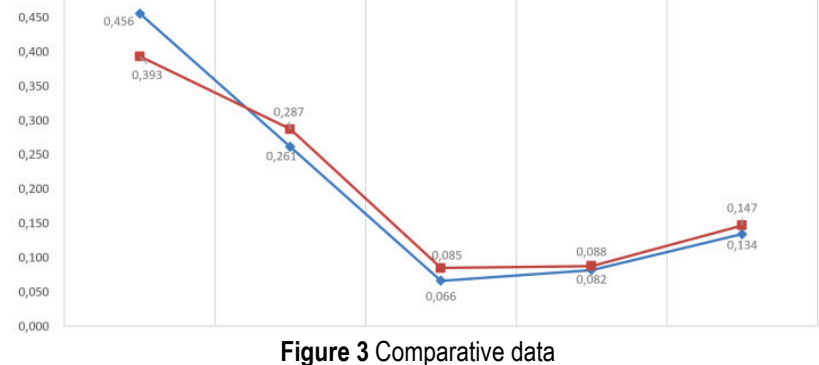

\section{CONCLUSION}

The motivation for the present work was to further investigate the c-credibility measure in the fuzzy environment and the possibility of its application in complex systems. The uncertainty in fuzzy systems is treated in a new manner. The c-credibility in relation to the aggregation function $h$ of fuzzy events is used in the ranking process. In the fuzzy environment this measure allows users to choose different aggregation functions depending on the observed problem and that will be the goal in some other research.

Simulated variables ranked by c-measure credibility, allows minimizing the subjectivity of expert judgment by fuzzy logic, and in some circumstances generating a better solution compared to others. Compared to the results obtained by another model, the result of this model shows a slight correction in the value of the most influential parameters, which could be attributed to the use of a credibility measure.

It is expected that the proposed procedure, which is implemented in the fuzzy logic model, will assist professionals in solving various problems and improve their knowledge of both logistics systems and other complex systems, especially on the level of simulation where they can choose different parameters in relatively simple and fast way.

\section{Acknowledgement}

The second author acknowledges the financial support of the Ministry of Education, Science and Technological Development of the Republic of Serbia, in the frame of Projects applied under No. TR 34014 and No. ON 174009.

\section{REFERENCES}

[1] Dubois, D. H. \& Prade, H. (2004). On the use of aggregation operations in information fusion processes. Fuzzy Sets and Systems, 142, 143-161. https://doi.org/10.1016/j.fss.2003.10.038

[2] Errampalli, M., Okushima, M., \& Akiyama T. (2013). Development of the Microscopic Traffic Simulation Model with the Fuzzy Logic technique. SIMULATION, 89(1), 87101. https://doi.org/10.1177/0037549712443716

[3] Gajovic, V., Kerkez, M., \& Kocovic, J. (2017). Modeling and simulation of logistic processes: risk assessment with a fuzzy logic technique. Simulation. Transactions of the Society for Modeling and Simulation International, 1-12. https://doi.org/10.1177/0037549717738351

[4] Grabish, M., Marichal, J-L, Mesiar, R., \& Pap, E. (2009). Aggregation Functions, Cambridge University Press, Cambridge. https://doi.org/10.1017/CBO9781139644150

[5] Klir, G. J. \& Yuan, B. (1995). Fuzzy sets and fuzzy logic, theory and applications, Prentice Hall, New Jersey.

[6] Koissi, M-C. \& Shapiro, A. F. (2012). Credibility Theory in a Fuzzy Environment, ARCH 2013.1 Proceedings August 14.

[7] Li, X. \& Liu, X. B. (2006). A sufficient and necessary condition for credibility measures, International Journal of Uncertainty. Fuzziness \& Knowledge-Based Systems, 14, 527-535. https://doi.org/10.1142/S0218488506004175

[8] Liu, B. (2007). Uncertainty Theory, $2^{\text {nd }}$ ed., Springer-Verlag, Berlin.

[9] Liu, B. \& Liu, Y. K. (2002). Expected Value of Fuzzy Variable and Fuzzy Expected Value Models. IEEE Transactions on Fuzzy Systems, 10, 445-450. https://doi.org/10.1109/TFUZZ.2002.800692

[10] Paunović, M, Ralević, N. M., Gajović, V., MladenovićVojinović, B., \& Milutinović, O. (2018). Two-Stage Fuzzy Logic Model for Cloud Service Supplier Selection and Evaluation, Mathematical Problems in Engineering, volume 2018, 11 pages, Hindawi Publishing Corporation. https://doi.org/10.1155/2018/7283127

[11] Pourrahmani, E., Delavar, M., \& Mir Abolfazl Mostafavi, M. A. (2015). Optimization of an evacuation plan with uncertain demands using fuzzy credibility theory and genetic algorithm. International Journal of Disaster Risk Reduction, 14(4), 357-372. https://doi.org/10.1016/j.ijdrr.2015.09.002

[12] Ralevic, N. M. \& Paunovic, M. (2019). c-Credibility Measure. FILOMAT, 33(9), 2571-2582. https://doi.org/10.2298/FIL1909571R

[13] Sii, S. H., Ruxton, T., \& Wang, J. (2001). A fuzzy-logicbased approach to quantitative safety modelling for marine systems. Reliability Engineering and System Safety, 73(1), 19-34. https://doi.org/10.1080/09617353.2001.11690715

[14] Wang, J., Lu, Z., \& Shi, Y. (2018). Aircraft icing safety analysis method in presence of fuzzy inputs and fuzzy state. Aerospace Science and Technology, Volumes 82-83, 172184. https://doi.org/10.1016/j.ast.2018.09.003

[15] Yu, W., Hou, G., Xia, P., \& Li, J. (2019). Supply Chain Joint Inventory Management and Cost Optimization Based on Ant Colony Algorithm and Fuzzy Model. Tehnički vjesnik, 26(6), 1729-1737. https://doi.org/10.17559/TV-20190805123158

[16] Zhang, S., Chen, M., Zhang, W., \& Zhuang, X. (2020). Fuzzy optimization model for electric vehicle routing problem with time windows and recharging stations. Expert Systems with Applications, 145, 113-123. https://doi.org/10.1016/j.eswa.2019.113123

Contact information:

Marija PAUNOVIĆ, PhD

(Corresponding author)

Faculty of Hotel Management and Tourism in Vrnjacka Banja,

University of Kragujevac, 36210 Vrnjacka Banja, Serbia

Email: majap@rcub.bg.ac.rs

Nebojša RALEVIĆ, Full Professor

Department of Fundamentals Sciences, Faculty of Technical Science,

University of Novi Sad, 21000 Novi Sad, Serbia

E-mail:nralevic@uns.ac.rs

Vladimir GAJOVIĆ, PhD

Dunav Insurance Company, 11000 Belgrade, Serbia

Email: vladimir.gajovic@dunav.com 